\title{
Interference of Notch 2 inhibits the progression of gliomas and induces cell apoptosis by induction of the cell cycle at the G0/G1 phase
}

\author{
HUI-PING YU ${ }^{1,2}$, SONG-TAO QI ${ }^{1}$, WEN-FENG FENG ${ }^{1}$, GUO-ZHONG ZHANG ${ }^{1}$, \\ HE-PING ZHANG ${ }^{2}$ and JIN-JUN TIAN ${ }^{2}$ \\ ${ }^{1}$ Department of Neurosurgery, Nanfang Hospital, Southern Medical University, Guangzhou, Guangdong 510515; \\ ${ }^{2}$ Department of Neurosurgery, The First Hospital of Quanzhou Affiliated to Fujian Medical University, \\ Quanzhou, Fujian 362000, P.R. China
}

Received January 20,2014; Accepted September 9, 2014

DOI: $10.3892 / \mathrm{mmr} .2014 .2747$

\begin{abstract}
Glioblastoma is the most common type of malignant brain tumor with a poor prognosis. The Notch signaling pathway is often aberrantly activated in glioma cells. In order to determine the expression of Notch 2 and to evaluate its possible prognostic value in malignant glioblastoma, specimens from 32 patients and 20 controls were analyzed using immunohistochemical staining and reverse transcription quantitative polymerase chain reaction. The expression of Notch 2 in the glioma tissues was significantly higher compared with that in the normal brain tissues $(\mathrm{P}<0.01)$. Subsequently, endogenous Notch 2 interference was effectively performed by specific small hairpin (sh)RNA in the glioma cancer cell line U251. The results from an MTT assay and from Annexin V-fluorescein isothiocyanate/propidium iodide staining indicated that interference of Notch 2 significantly inhibited the proliferation and induced the apoptosis of U251 cells. In addition, the cell cycle was analyzed using flow cytometry and the results revealed that Notch 2 shRNA induced cell cycle arrest at the G0/G1 phase in U251 cells. Additionally, proteins associated with the cell cycle and cell proliferation were detected using western blot analysis. The data demonstrated that the expression of P21, cyclin D and phosphorylated retinoblastoma was significantly inhibited in the Notch 2 shRNA-transfected U251 cells. The results of the present study provide further insights into the effects of Notch 2 and a molecular reference for brain tumor therapy.
\end{abstract}

Correspondence to: Professor Song-Tao Qi, Department of Neurosurgery, Nanfang Hospital, Southern Medical University, 1838 Guangzhou Road, Guangzhou, Guangdong 510515, P.R. China E-mail: qsongtaoqi@163.com

Key words: Notch 2, interference, gliomas, cell cycle

\section{Introduction}

A glioma is a type of malignant brain tumor that mainly arises from glial cells. The most common site of gliomas is in the brain (1). Gliomas represent $\sim 30 \%$ of tumors of the brain and central nervous system and $80 \%$ of malignant tumors in the brain (2). The main types of glioma are ependymomas, astrocytic gliomas, oligodendrogliomas, brainstem gliomas, optic nerve gliomas and mixed gliomas $(3,4)$. At present, due to the characteristics of glioma, including a rapid growth rate, unclear boundaries and frequent relapse following surgery, it remains one of the most intractable diseases in the field of neurosurgery (5). Therefore, it is particularly important to identify and develop new and effective treatment methods for glioma. Investigation of the targets of key molecules in the tumor signal transduction system has provided new hope for patients with gliomas.

The Notch signaling pathway is well known and is highly conserved in the majority of multicellular organisms (6). It has been confirmed that the occurrence of a variety of diseases, including cardiovascular disease and cancer, is closely associated with the abnormal activation of the Notch signaling pathway $(7,8)$. The abnormal activation of Notch is found in several types of cancer, including lung cancer(9), colon cancer (10), cervical cancer (11) and pancreatic cancer (12). Notch 2 is expressed in normal neuroblasts and controls neuronal differentiation (13). It is also one of the receptors with a single-pass transmembrane receptor protein (13). Notch signaling enhances the proliferative effects during neurogenesis in mammals $(14,15)$.

The present study aimed to clarify the effect of the Notch 2 protein on the proliferation of glioma cells. Initially, specimens from glioma tissues and normal brain tissues were obtained and the expression of Notch 2 was compared between them. Subsequently, RNA interference technology was used to knock down the expression of the Notch 2 protein in the human glioma cell line U251. Cell proliferation and cell cycle arrest were detected using an MTT assay and fluorescence-activated cell sorting (FACS). Additionally, proteins associated with the cell cycle and cell apoptosis 
were detected and compared using western blot analysis Therefore, the present study aimed to provide new information to assist in the therapy of human gliomas.

\section{Materials and methods}

Specimens. A total of 32 glioma tumor samples and 20 normal tissue samples were obtained by surgical resection of traumatized brain tissue following traumatic brain injury. All the specimens were obtained from the Institute of Neurosurgery, Nanfang Hospital Affiliated to Southern Medical University (Guangzhou, China). All the experiments were performed on patients in compliance with the Helsinki Declaration and study approval was obtained from the Ethics Committee of Nanfang Hospital Affiliated to Southern Medical University. The patients and their families were well informed of the details and written informed consent was obtained prior to the study.

Immunohistochemical staining. The specimens, obtained from the glioma tumor and normal brain tissues, were fixed in neutral buffered paraformaldehyde and processed for hematoxylin and eosin staining. The process of immunohistochemical staining was performed, as described previously $(16,17)$. The primary antibody used was rabbit polyclonal anti-Notch 2 (cat no. NB600-879; Novus Biologicals, Littleton, CO, USA), which was diluted (1:200) for use in the immunohistochemical analysis.

Reverse transcription quantitative polymerase chain reaction $(R T-q P C R)$. RT-qPCR was performed to detect the Notch 2 mRNA expression levels in the glioma and normal brain tissues. Firstly, RNA was reverse transcribed into cDNA and the resulting cDNA was used as templates for PCR amplification. The primer sequences were as follows: Upstream, 5'-ATGACTGCCCTAACCACAGG-3' and downstream, 5'-TGCAGTCATCTCCACTCCAG-3' for Notch 2; upstream, 5'-AGAGCTACGAGCTGCCTGAC-3' and downstream, 5'-AGCACTGTGTTGGCGTACAG-3' for $\beta$-actin. Here, $\beta$-actin was used as the internal control. A PCR kit (Invitrogen Life Technologies) was used and the cycle conditions were an initial step (hot start) of $95^{\circ} \mathrm{C}$ for $10 \mathrm{~min}$ prior to amplification cycles, followed by the PCR conditions of denaturation $\left(95^{\circ} \mathrm{C}\right.$ for $15 \mathrm{sec})$, annealing $\left(59^{\circ} \mathrm{C}\right.$ for $\left.20 \mathrm{sec}\right)$ and elongation $\left(72^{\circ} \mathrm{C}\right.$ for $15 \mathrm{sec}$ ) for a total of 25 cycles.

Cell line and short hairpin (sh)RNA. The human glioma cell line U251 was cultured in Dulbecco's modified Eagle's medium containing $10 \%$ fetal calf serum (Hyclone Laboratories, Inc., Logan, UT, USA), penicillin and streptomycin $(100 \mathrm{U} / \mathrm{ml}$; Gibco-BRL, Carlsbad, CA, USA) at $37^{\circ} \mathrm{C}$ in $5 \% \mathrm{CO}_{2}$. Notch 2 shRNA (human) was obtained from Santa Cruz Biotechnology, Inc. (Santa Cruz, CA, USA; cat no. sc-40135-V) and the control shRNA of Lentiviral Particles-A was obtained from Santa Cruz Biotechnology, Inc.; cat no. sc-108080).

Western blot analysis. The U251 cells were seeded into 48-well plates. After 3 days, the whole-cell extracts were prepared and the proteins were separated by PAGE, as previously described (18-20).
MTT assay. An MTT assay was performed, as described

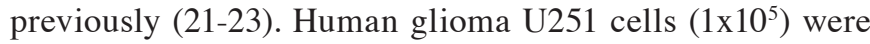
seeded into a 48-well plate. Following culture for different time periods (1-7 days), the plates were read on a microplate reader (Corning, Inc., Acton, MA, USA) with a test wavelength of $490 \mathrm{~nm}$.

Flow cytometric analysis. The apoptosis of human glioma U251 cells was determined using annexin V-fluorescein isothiocyanate (FITC)/propidium iodide (PI) dual staining according to the manufacturer's instructions (Santa Cruz Biotechnology, Inc.) and cell cycle analysis was performed using PI staining, as described previously $(24,25)$. Cells were then subjected to FACS analysis and $>10,000$ events were recorded in every example. The experiment was performed at least three independent times.

Statistical analysis. Basic statistical analyses were performed using the statistical software, SPSS 18.0 (SPSS, Inc., Chicago, IL, USA). All results are expressed as the mean \pm standard deviation. $\mathrm{P}<0.01$ was considered to indicate a statistically significant difference.

\section{Results}

Positive Notch 2 staining is observed in the majority of gliomas. In order to confirm the expression of Notch 2 in gliomas and in normal brain tissues, immunohistochemical staining analysis was performed. As shown in Fig. 1A-D, positive staining for Notch 2 appeared as brown granules, which were predominantly located in the cell membrane, nucleus and cytoplasm. However, only a small quantity of visible staining, indicating expression of Notch 2, was observed in normal brain tissues.

Subsequently, RT-qPCR was performed to further confirm the expression of Notch 2 in the glioma and normal brain tissues. As shown in Fig. 1E, the expression of Notch 2 in the glioma tissues was increased significantly compared with the normal brain tissues $(\mathrm{P}<0.01)$.

Notch 2 shRNA decreases the expression of Notch 2 in U251 cells. Notch 2 shRNA was used for interference of endogenous Notch 2 gene expression. The silencing effect was then determined using RT-qPCR. As shown in Fig. 2, the Notch 2 shRNA effectively interfered with the expression of Notch 2. The control shRNA was used as a negative control.

Knocking down the expression of Notch 2 inhibits the growth of the glioma cell line U251. In order to determine whether Notch 2 knockdown inhibited the growth of U251 cells, an MTT assay was performed. As shown in Fig. 3, 4 days after transfection with shRNA, the optical density (OD)490 values in the U251 cells transfected with Notch 2 shRNA were lower compared with the control cells ( ${ }^{*} \mathrm{P}<0.05$ and $\left.{ }^{* *} \mathrm{P}<0.01\right)$. Additionally, inhibition of U251 cell proliferation occurred in a time-dependent manner.

Transfection of Notch 2 shRNA induces the apoptosis of U251 cells. The present study then aimed to assess whether treating cancer cells with Notch 2 shRNA further induced 


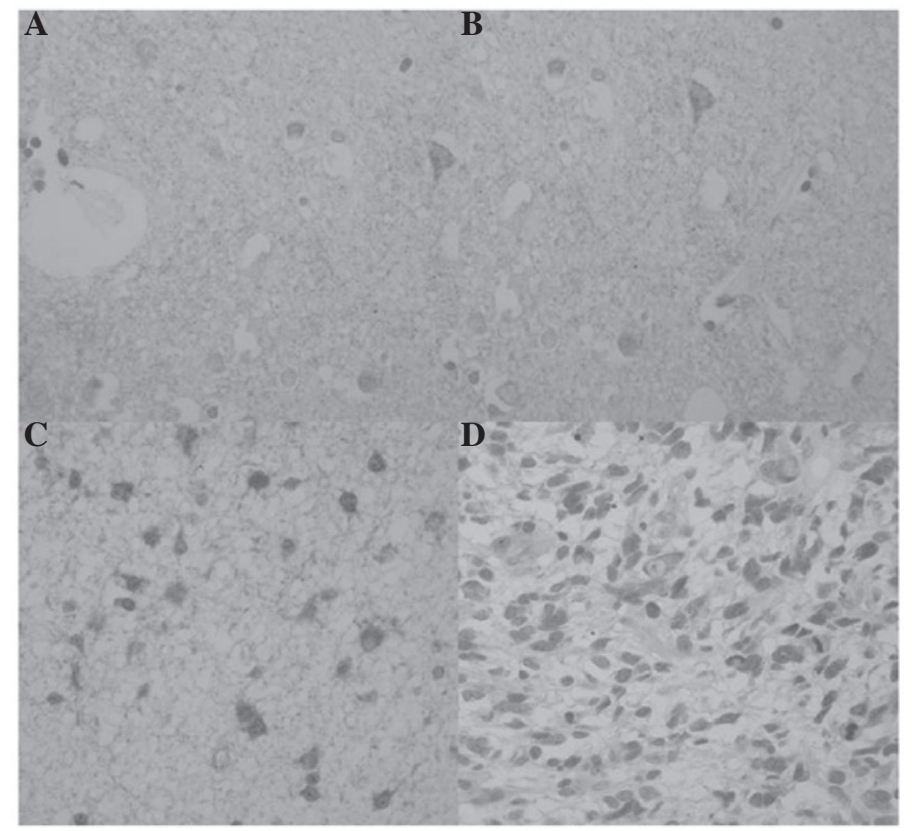

$\mathbf{E}$

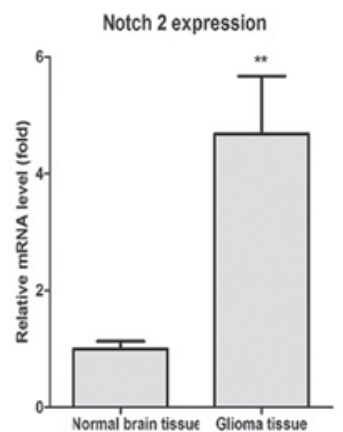

Figure 1. Paraffin-embedded samples were analyzed using histochemical staining for Notch 2. (A and B) Normal expression of Notch 2 was detected in the normal brain tissues and images were captured (magnification, $\mathrm{x} 400$ ). (D) Expression of Notch 2 was detected in the glioma tissues and images were captured (magnification, $\mathrm{x} 400$ ). (E) Total RNA was extracted and the expression of Notch 2 was assessed using reverse transcription quantitative polymerase chain reaction in the normal brain tissues and glioma tissues. ${ }^{* *} \mathrm{P}<0.01$, compared with normal brain tissues.

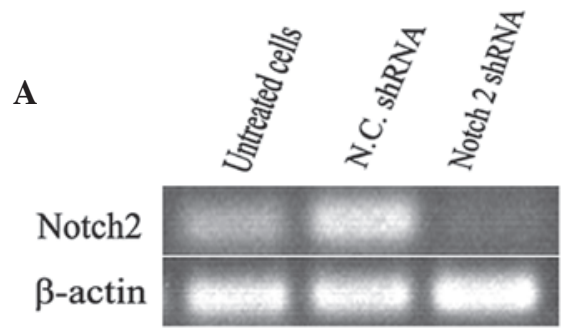

B



Figure 2. Expression of Notch 2 is effectively suppressed. The U251 cells were plated in 6-well plates. After $8 \mathrm{~h}$, Notch 2 shRNA or NC shRNA was transfected into the U251 cells. After $48 \mathrm{~h}$, total RNA was extracted and reverse transcribed into cDNA. Reverse transcription quantitative polymerase chain reaction was used to detect the expression of Notch 2. $\beta$-actin was used an internal reference. shRNA, short hairpin RNA; NC, negative control. "P<0.05, compared with untreated cells.

apoptosis. As shown in Fig. 4, Annexin V-FITC/PI dual staining was used to detect the apoptotic rate of the U251 cells. After 5 days, the results demonstrated that the apoptotic

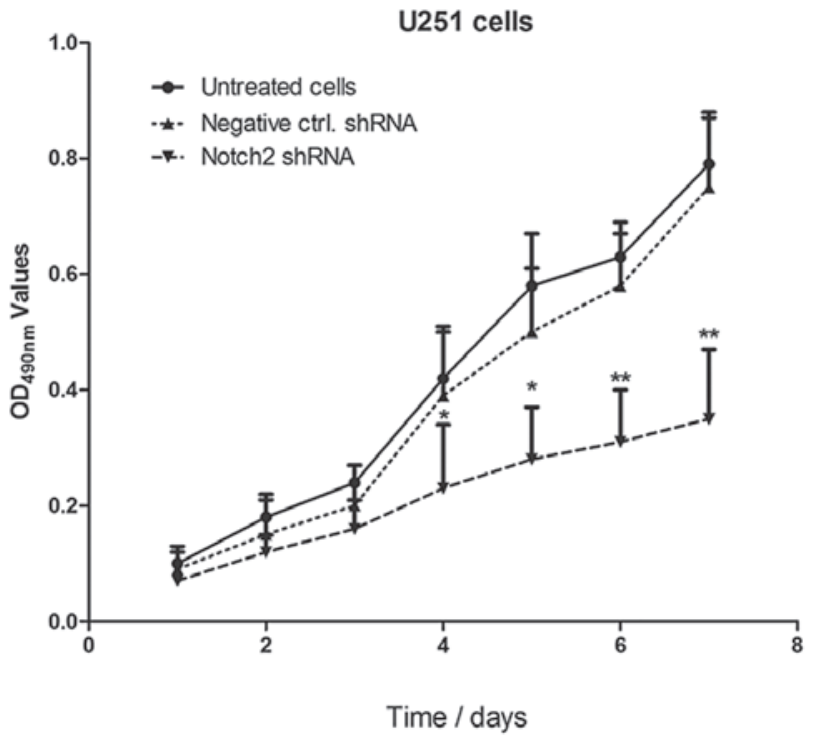

Figure 3. Interference of Notch 2 inhibits the growth of the glioma cell line U251. U251 cells were plated in 48-well plates and transfected with Notch 2 shRNA. The cells were then cultured for 7 days and an MTT assay was performed to detect cell viability. ${ }^{*} \mathrm{P}<0.05,{ }^{* * *} \mathrm{P}<0.01$, compared with the untreated cells. shRNA, short hairpin RNA; ctrl, control; OD, optical density.

rate of the cells transfected with Notch 2 shRNA was significantly higher in the Notch 2 shRNA group compared with the negative control shRNA group $(36.7 \pm 4.5 \%$ and $6.8 \pm 2.8 \%$, respectively; $\mathrm{n}=5 ; \mathrm{P}=0.0027$ ).

Notch 2 shRNA1 induces cell cycle arrest at the G0/G1 phase in U251 cells. Subsequently, cell cycle distribution was assessed in the different groups of cells. As shown in Fig. 5, 
A
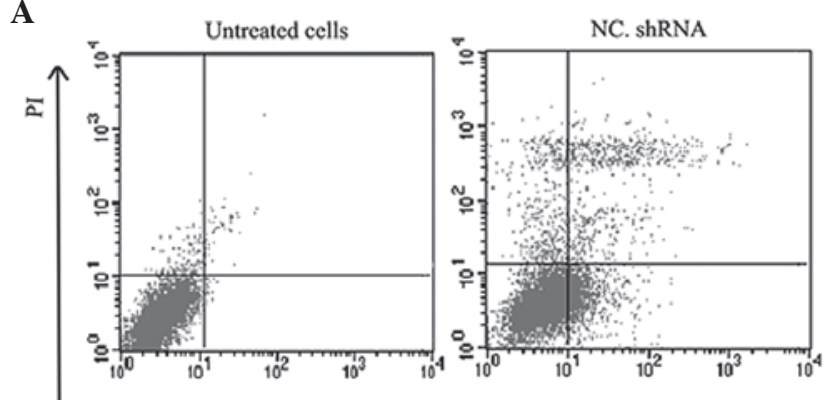

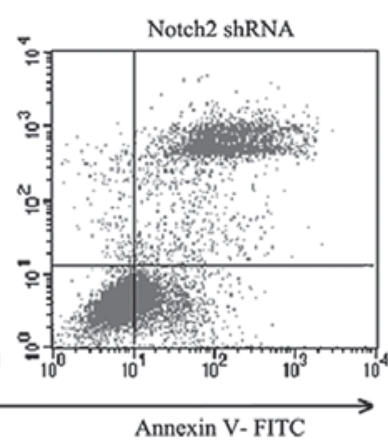

B



Figure 4. Transfection of Notch 2 shRNA induces the apoptosis of U251 cells (A) U251 cells were plated into a 6-well plate and transfected with Notch 2 shRNA. After 3 days, cell apoptosis was detected using Annexin V-FITC/PI staining. (B) Histogram of the cell apoptotic rate. Untreated cells or cells transfected with NC shRNA were used as negative controls. ${ }^{* *} \mathrm{P}<0.01$, compared with the untreated cells. FITC, fluorescein isothiocyanate; PI, propidium iodide; shRNA, small hairpin RNA; NC, negative control.

\section{Cell distribution of U251 cells}

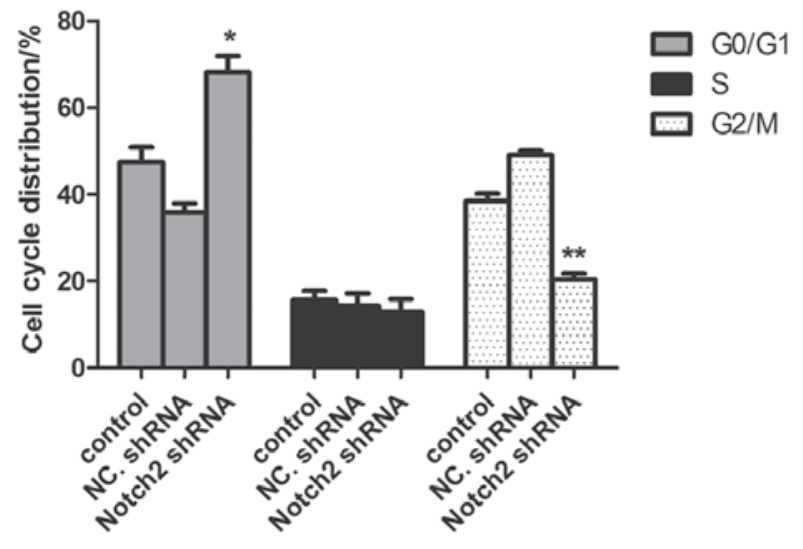

Different Groups

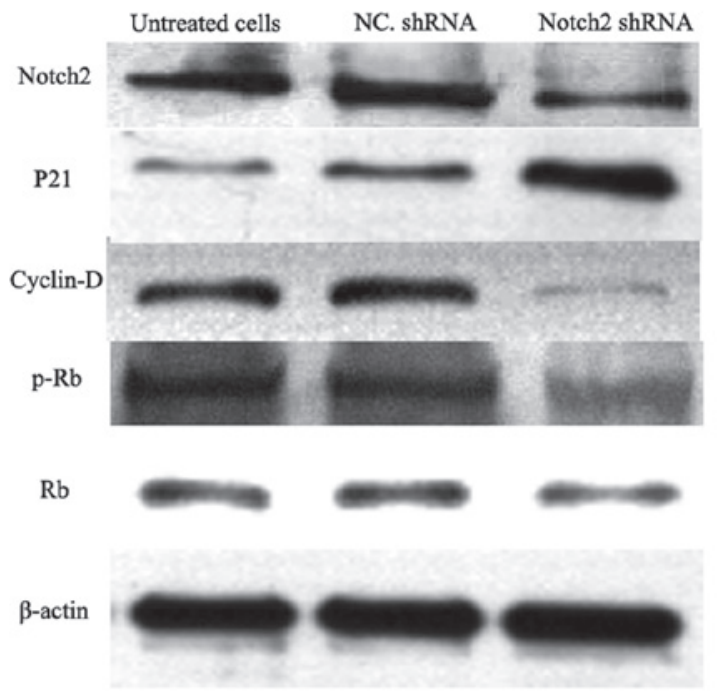

Figure 6. Notch 2 shRNA affects the expression of proteins associated with the cell cycle. Cell lysates were prepared and the expression of P21, cyclin D1 and $\mathrm{p}-\mathrm{Rb}$ were detected using western blot analysis. $\beta$-actin was used as an internal reference. The untreated cells and the cells transfected with negative control shRNA were used as negative controls. $\mathrm{Rb}$, retinoblastoma; $\mathrm{p}-\mathrm{Rb}$, phosphorylated retinoblastoma; shRNA, short hairpin RNA; NC, negative control.

\section{Discussion}

Gliomas are the most common and malignant type of tumor in the central nervous system (26). Glioma is particularly difficult to treat, with high rates of recurrence and low median survival rates. However, the treatment of gliomas is constantly improving and combination therapy, surgical and concurrent radiotherapy and chemotherapy are used. Despite this, the rate of recurrence is almost $100 \%(27,28)$, with little improvement in efficacy and a poor prognosis. The present study demonstrated that the Notch 2 protein exhibited abnormal and high expression of the Notch 2 protein in human glioma tissues. Following interference of Notch 2, the proliferation of the glioma cell line U251 was significantly inhibited, the cell cycle was arrested at the G0/G1 phase and apoptotic rates were markedly increased. These results suggested that the Notch 2 
protein may be an effective target molecule in the therapy of gliomas. Additionally, it is key in several important pathways in tumor development and progression.

Purow et al (8) successfully reduced the expression of Notch 1 protein in the human glioma cell line U251 using RNA interference technology. The cells were then implanted into nude mice, which significantly prolonged the survival rate of the mice compared with the control group. Several studies have also demonstrated that activation of the Notch signaling pathway can stimulate cell proliferation in acute $\mathrm{T}$ lymphoblastic leukemia, breast cancer, renal epithelial tumor with transitional cells and pancreatic cancer (12,29-33). In the present study, the effects of depletion of Notch 2 by RNA interference on glioma cell cycle progression was investigated. The results revealed that loss of Notch 2 led to cell cycle arrest at the G0/G1 phase in U251 cells, with a significant decrease in the proportion of $\mathrm{U} 251$ cells in the $\mathrm{G} 2 / \mathrm{M}$ phase. In addition, cell cycle arrest at the G0/G1 phase was accompanied by an accumulation of p21 and a decrease in cyclin $\mathrm{D}$ and $\mathrm{p}-\mathrm{Rb}$. All these results were consistent with each other.

In conclusion, the results of the present study demonstrated that interference of Notch 2 may provide a useful therapeutic approach in the treatment of human brain tumors and, to a certain extent, provides theoretical support for the use of gene therapy in the treatment of human gliomas.

\section{References}

1. Mamelak AN and Jacoby DB: Targeted delivery of antitumoral therapy to glioma and other malignancies with synthetic chlorotoxin (TM-601). Expert Opin Drug Deliv 4: 175-186, 2007.

2. Goodenberger ML and Jenkins RB: Genetics of adult glioma. Cancer Genet 205: 613-621, 2012.

3. Tobias A, Ahmed A, Moon KS and Lesniak MS: The art of gene therapy for glioma: a review of the challenging road to the bedside. J Neurol Neurosurg Psychiatry 84: 213-222, 2013.

4. Carpentier AF, Auf G and Delattre JY: CpG-oligonucleotides for cancer immunotherapy: review of the literature and potential applications in malignant glioma. Front Biosci 8: e115127, 2003

5. Gotz I and Grosu AL: ([18)F]FET-PET Imaging for treatment and response monitoring of radiation therapy in malignant glioma patients - a review. Front Oncol 3: 104, 2013.

6. Artavanis-Tsakonas S, Rand MD and Lake RJ: Notch signaling: cell fate control and signal integration in development Science 284: 770-776, 1999.

7. Cao Q, Lu J, Kaur C, et al: Expression of Notch-1 receptor and its ligands Jagged-1 and Delta-1 in amoeboid microglia in postnatal rat brain and murine BV-2 cells. Glia 56: 1224-1237, 2008.

8. Purow BW, Haque RM, Noel MW, et al: Expression of Notch-1 and its ligands, Delta-like-1 and Jagged-1, is critical for glioma cell survival and proliferation. Cancer Res 65: 2353-2363, 2005.

9. Baumgart A, Mazur PK, Anton M, Rudelius M, Schwamborn K, Feuchtinger A, et al: Opposing role of Notch1 and Notch2 in a KrasG12D-driven murine non-small cell lung cancer model. Oncogene: Feb 10, 2014 (Epub ahead of print). doi: 10.1038/ onc.2013.592.

10. Bertrand FE, Angus CW, Partis WJ and Sigounas G: Developmental pathways in colon cancer: crosstalk between WNT, BMP, Hedgehog and Notch. Cell Cycle 11: 4344-4351, 2012.

11. Zhang P, Li H, Yang B, Yang F, Zhang LL, Kong QY, et al: Biological significance and therapeutic implication of resveratrol-inhibited Wnt, Notch and STAT3 signaling in cervical cancer cells. Genes Cancer 5: 154-164, 2014.
12. Ristorcelli E and Lombardo D: Targeting Notch signaling in pancreatic cancer. Expert Opin Ther Targets 14: 541-552, 2010.

13. Brou C, Logeat F, Gupta N, et al: A novel proteolytic cleavage involved in Notch signaling: the role of the disintegrin-metalloprotease TACE. Mol Cell 5: 207-216, 2000.

14. Hatakeyama J, Wakamatsu Y, Nagafuchi A, Kageyama R, Shigemoto R and Shimamura K: Cadherin-based adhesions in the apical endfoot are required for active Notch signaling to control neurogenesis in vertebrates. Development 141: 1671-1682, 2014.

15. Vilas-Boas F, Fior R, Swedlow JR, Storey KG and Henrique D: A novel reporter of notch signalling indicates regulated and random Notch activation during vertebrate neurogenesis. BMC Biol 9: 58, 2011.

16. Saleem M, Maddodi N, Abu Zaid M, et al: Lupeol inhibits growth of highly aggressive human metastatic melanoma cells in vitro and in vivo by inducing apoptosis. Clin Cancer Res 14: 2119-2127, 2008.

17. Adhami VM, Siddiqui IA, Ahmad N, Gupta S and Mukhtar H: Oral consumption of green tea polyphenols inhibits insulin-like growth factor-I-induced signaling in an autochthonous mouse model of prostate cancer. Cancer Res 64: 8715-8722, 2004.

18. Nishitani H, Sugimoto N, Roukos V, et al: Two E3 ubiquitin ligases, SCF-Skp2 and DDB1-Cul4, target human Cdt1 for proteolysis. EMBO J 25: 1126-1136, 2006.

19. Peng L, Xu Z, Zhou Y, Yang T, Liang ZQ and Zhang M: Effect of rosiglitazone on cells cycle, apoptosis and expression of Skp2 and p27Kip1 in hepatocellular carcinoma cell line. Zhonghua Gan Zang Bing Za Zhi 18: 148-149, 2010 (In Chinese).

20. Schulman BA, Carrano AC, Jeffrey PD, et al: Insights into SCF ubiquitin ligases from the structure of the Skp1-Skp2 complex. Nature 408: 381-386, 2000.

21. Li Y, Huang W, Huang S, Du J and Huang C: Screening of anti-cancer agent using zebrafish: comparison with the MTT assay. Biochem Biophys Res Commun 422: 85-90, 2012.

22. Sarzaeem A, Zare Mirakabadi A, Moradhaseli S, Morovvati H and Lotfi M: Cytotoxic effect of ICD-85 (venom-derived peptides) on HeLa cancer cell line and normal LK cells using MTT assay. Arch Iran Med 15: 696-701, 2012

23. Sylvester PW: Optimization of the tetrazolium dye (MTT) colorimetric assay for cellular growth and viability. Methods Mol Biol 716: 157-168, 2011.

24. Guo LD, Chen XJ, Hu YH, Yu ZJ, Wang D and Liu JZ: Curcumin inhibits proliferation and induces apoptosis of human colorectal cancer cells by activating the mitochondria apoptotic pathway. Phytother Res 27: 422-430, 2013.

25. Zhang CJ, Gu LG and Yu HT: Antagonism of baicalin on cell cyclical distribution and cell apoptosis in A549 cells infected with influenza A (H1N1) virus. Bing Du Xue Bao 27: 108-116, 2011 (In Chinese).

26. You G, Yan W, Zhang W, Li S, Li G and Jiang T: Isolated angiitis of the central nervous system with tumor-like lesion, mimicking brain malignant glioma: a case report and review of the literature. World J Surg Oncol 9: 97, 2011.

27. Lakomý R, Fadrus P, Slampa P, et al: Multimodal treatment of glioblastoma multiforme: results of 86 consecutive patients diagnosed in period 2003-2009. Klin Onkol 24: 112-120, 2011 (In Czech).

28. Sharma DN, Goyal SG, Muzumder S, et al: Radiation therapy in paediatric gliomas: our institutional experience. Neurol Neurochir Pol 44: 28-34, 2010.

29. Palomero T and Ferrando A: Therapeutic targeting of NOTCH1 signaling in T-cell acute lymphoblastic leukemia. Clin Lymphoma Myeloma 9: S205-S210, 2009

30. Zardawi SJ, O'Toole SA, Sutherland RL and Musgrove EA Dysregulation of Hedgehog, Wnt and Notch signalling pathways in breast cancer. Histol Histopathol 24: 385-398, 2009.

31. Sjolund J, Johansson M, Manna S, et al: Suppression of renal cell carcinoma growth by inhibition of Notch signaling in vitro and in vivo. J Clin Invest 118: 217-228, 2008.

32. Dai J, Ma D, Zang S, et al: Cross-talk between Notch and EGFR signaling in human breast cancer cells. Cancer Invest 27: 533-540, 2009.

33. Yen WC, Fischer MM, Hynes M, et al: Anti-DLL4 has broad spectrum activity in pancreatic cancer dependent on targeting DLL4-Notch signaling in both tumor and vasculature cells. Clin Cancer Res 18: 5374-5386, 2012. 